\title{
Efecto del Glifosato sobre el CRecimiento y ACumulación de AZÚCARES LIBRES EN DOS BIOTIPOS DE Lolium perenne DE DisTINTA SENSIBILIDAD AL HERBICIDA ${ }^{1}$
}

\author{
Glyphosate Effects on the Growth and Free Sugar Accumulation of Two Lolium perenne \\ Biotypes with Different Herbicide Sensitivity
}

\begin{abstract}
YANNICCARI, M. ${ }^{2}$, ISTILART, C. ${ }^{3}$, GIMÉNEZ, D.O. ${ }^{4}$, ACCIARESI, H. ${ }^{5}$ y CASTRO, A.M. ${ }^{6}$
RESUMEN - El movimiento sistémico del glifosato está determinado por el transporte de fotoasimilados. A su vez, la capacidad de un destino de consumir los asimilados está condicionada por su actividad metabólica. Pese a su importancia, la relación entre el glifosato y la síntesis de azúcares en hojas fuente ha sido poco abordada. El objetivo del presente trabajo fue evaluar los efectos del glifosato sobre el crecimiento y la acumulación de azúcares libres en dos biotipos de Lolium perenne de baja y alta sensibilidad al herbicida. Se trabajó con clones de ambos tipos de plantas, en macollaje, tratados con 1.440 g e.a. ha ${ }^{-1}$ de glifosato y sin tratamiento herbicida como controles. Se evaluó periódicamente el efecto del glifosato sobre el rebrote de hojas hasta las 50 horas post-aplicación y sobre los niveles de azúcares libres totales, reductores y no reductores en hojas a 1, 2, 3 y 5 días post-aplicación. A partir de las 25 horas post-aplicación, el glifosato provocó una disminución del crecimiento del $58 \%$ en el biotipo susceptible, con una acumulación de azúcares libres superior al $90 \%$ con relación al control, desde el primer día post-aplicación en adelante. La inhibición del crecimiento, inducida por el glifosato en plantas susceptibles, no depende de la limitación del traslado de fotoasimilados desde la parte aérea. Por tanto, la acumulación de azúcares libres en hojas podría explicarse por la caída en la tasa de crecimiento. En el biotipo de baja sensibilidad, en el que no se detectó inhibición del crecimiento, estos efectos fueron limitados.
\end{abstract}

Palabras-clave: resistencia a herbicidas, Ryegrass Perenne, fotoasimilados.

\begin{abstract}
The systemic movement of glyphosate is determined by the transport of photoassimilates. In turn, the capacity of a destination to consume assimilates is conditioned by their metabolic activity. Despite its importance, the relationship between the glyphosate and the sugar synthesis from source leaves has been little studied. The aim of this work was to determine the effect of glyphosate on the growth and free sugar accumulation of two Lolium perenne biotypes of low and high glyphosate sensitivity. It was worked with clones of both types, in tillering, sprayed with 1,440 g a.e. ha-1 of glyphosate as treatments and without herbicide as controls. The glyphosate effects on the regrowth of leaves was studied until 50 hours post-application and on total free sugar, reducing free sugar and nonreducing free sugar from leaves to 1, 2, 3 and 5 days post-application were periodically evaluated. From 25 hours after glyphosate application, it caused on the susceptible biotype a growth decrease of 58\% and an accumulation of free sugar above $90 \%$ compared to controls. In susceptible biotypes, growth inhibition does not depend on a reduced photoassimilate translocation from the overground part. Therefore, the free sugar accumulation in leaves could be explained by the fall in the rate of growth. These effects are limited in the low sensitivity biotype, where growth inhibition has not been detected.
\end{abstract}

Keywords: herbicide resistance, perennial rye grass, photoassimilates.

1 Recebido para publicação em 28.7.2011 e aprovado em 27.2.2012.

2 Ing. Agr., Doctorando, Instituto de Fisiología Vegetal, Universidad Nacional de La Plata (UNLP), Consejo Nacional de Investigaciones Científicas y Técnicas (CONICET), < marcosyanniccari@conicet.gov.ar>; ${ }^{3}$ Ing. Agr., Chacra Experimental Integrada Barrow, Instituto Nacional de Tecnología Agropecuaria, Ministerio de Asuntos Agrarios; ${ }^{4}$ Ing. Agr., Profesor de la Facultad de Cs. Agrarias y Forestales, UNLP; ${ }^{5}$ Ing. Agr., Dr., Profesor de la Facultad de Cs. Agrarias y Forestales, UNLP, Investigador CICBA; ${ }^{6}$ Ing. Agr., Dra., Profesora de la Facultad de Cs. Agrarias y Forestales, UNLP, Investigadora CONICET.

Planta Daninha, Viçosa-MG, v. 30, n. 1, p. 155-164, 2012 


\section{INTRODUCCIÓN}

El glifosato es el herbicida más empleado en el mundo (Woodburn, 2000) y junto con otros principios activos resulta ser uno de los insumos más demandados para el control de malas hierbas (Yamada et al., 2009). Se ha demostrado que la aplicación continua de glifosato, durante al menos diez años y asociada a la reducida rotación de cultivos, ha provocado cambios en las comunidades de malas hierbas. En ciertos casos, esos cambios florísticos han llevado a la aparición de biotipos de baja sensibilidad al herbicida (Owen, 2008).

En ese sentido, en Sudamérica han sido reportados numerosos casos de poblaciones de malezas de alta diversidad taxonómica resistentes a glifosato (Vila-Aiub et al., 2008). Particularmente, dentro del género Lolium se han detectado poblaciones de Lolium multiflorum resistentes a glifosato en Chile (Perez \& Kogan, 2003), Brasil (Roman et al., 2004; Vargas et al., 2004) y Argentina (Vigna et al., 2007). Además, en este último país, se ha comunicado la resistencia a glifosato de una población de Lolium perenne L. (Yanniccari et al., 2008). La evolución de la resistencia ha tenido lugar en agroecosistemas donde el glifosato ejerce una fuerte y continua presión de selección (Vila-Aiub et al., 2008).

La acción fitotóxica del glifosato se debe a la inhibición de la enzima 5enolpiruvilshikimato-3-fosfato sintasa (EPSPs), responsable por catalizar la reacción entre el shikimato-3-fosfato y el fosfoenolpiruvato (Schönbrunn et al., 2002). Entre las alteraciones provocadas por el herbicida, se ha verificado el incremento de los niveles de ácido shikímico (Pline et al. 2002), la disminución del pool de aminoácidos aromáticos (Franz et al., 1997), la inhibición de la regeneración de ribulosa-1,5-bifosfato, un metabolito clave del ciclo de Calvin (Servaites et al., 1987), y la reducción de la tasa de fotosintesis neta (Ribeiro et al., 2008; Olesen $\&$ Cedergreen, 2010).

Dentro de las particularidades del glifosato, como potente herbicida, se destaca la capacidad de ser trasladado hacia tejidos meristemáticos y órganos subterráneos (Shaner, 2009). El movimiento sistémico del herbicida hacia los destinos de activo crecimiento depende del transporte de fotoasimilados (Bromilow et al., 1990; Devine $\&$ Hall, 1990). Pese a su importancia, la relación entre el glifosato y la síntesis de azúcares en las hojas fuente ha sido poco abordada. Geiger y Bestman (1990) han estudiado el efecto del glifosato sobre el metabolismo del carbono, sosteniendo que las alteraciones provocadas inhiben el traslado de asimilados y, conjuntamente, del herbicida. Trabajando con materiales de Beta vulgaris de distinta sensibilidad al glifosato, Geiger et al. (1999) demostraron que en plantas resistentes no hay reflejos de alteraciones en el metabolismo del carbono ni en el traslado de asimilados, a diferencia de lo que sucede en plantas susceptibles.

La capacidad de un destino de consumir asimilados, determinado por su actividad metabólica, condiciona la demanda de carbohidratos (Mengel \& Kirkby, 2001). En este sentido, varios autores han encontrado en distintas especies una inhibición de la división celular provocada por el glifosato (Pline et al., 2002; El-Tayeb \& Zaki, 2009). Sin embargo, no está totalmente dilucidado si los efectos del herbicida sobre el menor traslado de asimilados se debe a la inhibición de la demanda de los tejidos en activo crecimiento, o si la reducción en la actividad metabólica es una consecuencia de un limitado transporte de carbohidratos desde las hojas fuente producto de la acción del glifosato.

El objetivo del trabajo fue evaluar los efectos provocados por el glifosato sobre el crecimiento y la acumulación de azúcares libres totales, reductores y no reductores, en biotipos de Lolium perenne de baja y alta sensibilidad al herbicida.

La hipótesis de trabajo planteada sostiene que el glifosato inhibe el crecimiento de hojas en expansión en el biotipo susceptible, independientemente de las alteraciones provocadas en el metabolismo de azúcares en las hojas fuente, mientras que en el biotipo resistente, el herbicida no provoca alteraciones significativas en el crecimiento $y$, consecuentemente, el efecto sobre la acumulación de azúcares libres es menor que en las plantas altamente sensibles. 


\section{MATERIAL Y MÉTODOS}

\section{Material vegetal}

Fueron identificados dos biotipos, uno susceptible y otro resistente, caracterizados según la metodología de Baerson et al. (2002), a partir de la descendencia de una única planta de Lolium perenne proveniente de una población del sur de la provincia de Buenos Aires, Argentina. Este material fue identificado en campo y en laboratorio por su baja sensibilidad al glifosato (Yanniccari et al., 2011). Dichos biotipos fueron multiplicados vegetativamente por división de macollos, a fin de obtener el número de réplicas necesario para cada experimento que se describirá posteriormente.

Las plantas fueron cultivadas en invernadero, en macetas de $500 \mathrm{~cm}^{3}$, con tierra tamizada y mantenidas a capacidad de campo antes y durante los experimentos.

\section{Efectos del glifosato sobre el crecimiento}

Se trabajó con clones en pleno macollaje, con cinco a siete macollos por planta. Un grupo de cinco plantas de cada biotipo fue asperjado con una dosis de glifosato (sal isopropilamina del N-fosfonometil glicina, RoundUp ${ }^{\circledR} 48 \%$ ) de $1.440 \mathrm{~g}$ equivalente ácido (e.a.) ha $\mathrm{h}^{-1}$ (volumen de aplicación 100 litros de agua bidestilada $\mathrm{ha}^{-1}$ ). Otro lote similar de plantas fue utilizado como control y tratado con agua bidestilada más el 0,01\% de Tween 20 (Anedra ${ }^{\circledR}$ ).

A las 3 horas de realizados los tratamientos, las plantas fueron cortadas a $2,5 \mathrm{~cm}$ del cuello o nudo de ahijamiento. Esto se realizó con el fin de independizar los efectos del herbicida sobre la fotosintesis y el traslado de asimilados desde la parte aérea, respecto a los efectos del glifosato sobre el crecimiento de hojas. Luego de efectuado el corte, se midió la longitud del rebrote de las hojas por sobre el corte a las 4, 6, 9, 25, 30 y 50 horas postaplicación (HPA), para estimar así la actividad de los meristemas intercalares de la base de la lámina y de la vaina. Se empleó un calibre Panorama S.S. ${ }^{\circledR}$ de $1 / 10 \mathrm{~mm}$ de precisión y se realizaron las mediciones para todos los macollos de cada planta. Luego, estos valores se promediaron para obtener un único valor por planta.

\section{Efectos sobre la acumulación de azúcares libres}

Se trataron veinte plantas con cinco macollos de cada uno de los dos biotipos con glifosato, $1.440 \mathrm{~g}$ equivalente ácido $\mathrm{ha}^{-1}$, bajo las mismas condiciones que el experimento anterior. Se utilizó como control un lote con otras veinte plantas de cada biotipo, a las que se aplicó agua bidestilada más Tween 20, como en el ensayo previo. Se cosecharon cinco plantas por cada tratamiento y biotipo; se tomaron $500 \mathrm{mg}$ de las últimas hojas expandidas a los 1, 2, 3 y 5 días post-aplicación (DPA). El material se conservó a $20^{\circ} \mathrm{C}$.

Preparación del extracto: A cada muestra se le agregó $5 \mathrm{~mL}$ de etanol (85\% v/v) y se calentó a $100{ }^{\circ} \mathrm{C}$ durante 5 minutos. Se extrajo el sobrenadante y se lo conservó. Luego, al tubo con la muestra se le agregó otros $5 \mathrm{~mL}$ de etanol y se calentó a $100{ }^{\circ} \mathrm{C}$ durante 5 minutos. Este proceso se repitió tres veces. A partir de la colección del sobrenadante se obtuvo un extracto que fue enrasado a $10 \mathrm{~mL}$ por evaporación del etanol a $80^{\circ} \mathrm{C}$.

Determinación de azúcares totales: Con el extracto de cada muestra se realizaron cuatro repeticiones de $250 \mu \mathrm{L}$ con cada una. Luego, se le agregó $1 \mathrm{~mL}$ de agua bidestilada y 0,5 mL de $\mathrm{HCl}(0,1 \mathrm{M})$ a cada repetición, se agitó suavemente y se llevó a $100{ }^{\circ} \mathrm{C}$ durante 5 minutos. Se dejó enfriar a temperatura ambiente; se incorporó $1 \mathrm{~mL}$ del reactivo cúprico $(1,2 \% \mathrm{p} / \mathrm{v}$ de tartrato de potasio y sodio; $2,4 \% \mathrm{p} / \mathrm{v}$ de $\mathrm{Na}_{2} \mathrm{CO}_{3} ; 0,4 \% \mathrm{p} / \mathrm{v}$ de $\mathrm{CuSO}_{4} .5 \mathrm{H}_{2} \mathrm{O}$; $1,2 \% \mathrm{p} / \mathrm{v}$ de $\mathrm{NaHCO}_{3}$ y $18 \% \mathrm{p} / \mathrm{v}$ de $\mathrm{Na}_{2} \mathrm{SO}_{4}$ ) y se calentó a $100{ }^{\circ} \mathrm{C}$ durante 10 minutos. Se dejó enfriar y se agregó $0,5 \mathrm{~mL}$ del reactivo arsenomolibdato $\left(5 \% \mathrm{p} / \mathrm{v}\right.$ de $\left(\mathrm{NH}_{4}\right)_{6} \mathrm{Mo}_{7} \mathrm{O}_{24} \cdot 4 \mathrm{H}_{2} \mathrm{O}$; $4,2 \% \mathrm{v} / \mathrm{v}$ de $\mathrm{H}_{2} \mathrm{SO}_{4}$ concentrado y $0,6 \% \mathrm{p} / \mathrm{v}$ de $\left.\mathrm{Na}_{2} \mathrm{HAsO}_{4} \cdot 7 \mathrm{H}_{2} \mathrm{O}\right)$ y se agitó suavemente durante 10 segundos. Finalmente, se enrasó a $25 \mathrm{~mL}$ con agua bidestilada y se midió la absorbancia a $520 \eta \mathrm{m}$, utilizando un espectrofotómetro Shimadzu ${ }^{\circledR}$ UV-160.

Determinación de azúcares reductores: Se realizaron cuatro repeticiones por muestra, tomando en cada una $250 \mu \mathrm{L}$ del extracto. Se le agregó $1 \mathrm{~mL}$ del reactivo cúprico y se calentó a $100{ }^{\circ} \mathrm{C}$ durante 10 minutos. Luego, se agregó $0,5 \mathrm{~mL}$ del reactivo arsenomolibdato y se agitó suavemente durante 10 segundos. Igual que 
con el resultado anterior, se enrasó a $25 \mathrm{~mL}$ con agua bidestilada y se midió la absorbancia a $520 \eta \mathrm{m}$, utilizando un espectrofotómetro Shimadzu ${ }^{\circledR}$ UV-160.

En ambas determinaciones se utilizó como blanco $250 \mu \mathrm{L}$ de agua bidestilada tratada según el correspondiente protocolo. La concentración de azúcares se estimó a partir de una curva estándar realizada con distintas concentraciones de sacarosa $\left(0\right.$ a $500 \mu \mathrm{g} \mathrm{mL} \mathrm{m}^{-1}$, B.D.H. $\left.{ }^{\circledR}\right)$. Finalmente, los valores de las cuatro repeticiones de cada muestra fueron promediados.

Estimación de azúcares no reductores: Los azúcares no reductores fueron estimados por diferencia entre la concentración de azúcares totales y azúcares reductores.

Análisis estadistico: Los datos obtenidos en ambos experimentos fueron sometidos a un análisis de varianza (ANOVA) para diseños multifactoriales. Las fuentes de variación fueron: biotipo (susceptible y resistente), tiempo post-aplicación (HPA o DPA), tratamiento (control y aplicación de glifosato) y sus interacciones dobles y triples. Se realizó el estudio de residuales para corroborar el cumplimiento de los supuestos del ANOVA. A partir de los análisis de la varianza, la separación de medias se realizó mediante la prueba de Fisher de diferencias minimas significativas $(p<0,05)$.

\section{RESULTADOS Y DISCUSIÓN}

\section{Efectos del glifosato sobre el crecimiento}

Se observaron diferencias significativas en el crecimiento entre ambos biotipos, entre los controles y los tratamientos con glifosato y en las distintas evaluaciones post-aplicación (Tabla 1). De igual forma, las interacciones resultaron altamente significativas, a excepción del biotipo x HPA, debido a la falta de diferencias en el biotipo resistente. Se detectó que las diferencias en el crecimiento de ambos biotipos están explicadas por las interacciones biotipo $\mathrm{x}$ tratamiento, HPA $\mathrm{x}$ tratamiento y por la interacción triple, con valores de probabilidad altamente significativos.

A las 25 HPA se registró la primera diferencia significativa en el biotipo susceptible entre plantas tratadas con glifosato y su control (Figura 1). En ese momento, el herbicida provocó una disminución del crecimiento del $58 \%$ respecto al testigo sin glifosato. Posteriormente, esta diferencia se mantuvo en forma proporcional, en la medida que continuó el crecimiento de las plantas y transcurrió el tiempo post-aplicación (Figura 1). En contraste, el biotipo resistente en ningún momento mostró diferencias significativas en el crecimiento que hayan sido provocadas por el herbicida (Figura 1).

A partir de estos resultados, se sugiere que la inhibición provocada sobre el crecimiento del biotipo sensible por el herbicida es independiente de los efectos que el glifosato induce sobre la parte aérea. En concordancia, la caída en la tasa de crecimiento podría explicarse por la inhibición en la sintesis de aminoácidos aromáticos (Franz et al., 1997) que interferirian con la sintesis de enzimas necesarias en el metabolismo de los tejidos meristemáticos (Geiger \& Bestman, 1990).

Al realizar el corte de la parte aérea, luego de la aplicación de glifosato, se eliminó el efecto del herbicida sobre la fijación de $\mathrm{CO}_{2}$ y traslado de carbohidratos. En estas condiciones, se detectaron diferencias en el crecimiento entre plantas sensibles tratadas con el herbicida y sus controles.

Así, se demuestra que en el tiempo postaplicación evaluado, la inhibición del crecimiento es independiente de la inhibición de la fotosintesis y del traslado de fotoasimilados. Estos resultados se corresponden con los de El-Tayeb \& Zaki (2009), que demostraron el efecto inhibitorio del glifosato sobre la división celular de tejidos meristemáticos de raicillas de semillas en germinación de Vicia faba, independiente de los efectos del herbicida sobre la fotosintesis. Previamente, esta inhibición había sido observada sobre semillas en germinación de Lolium spp. (Perez Jones et al., 2007).

El crecimiento en el biotipo resistente no es inhibido por efecto del glifosato; tanto los mecanismos dependientes de la enzima blanco (Wakelin \& Preston, 2006) como la limitación del traslado del herbicida podrian estar puestos en juego (Shaner, 2009). 
Tabla 1 - Efectos del biotipo (susceptible y resistente); horas post-aplicación (4, 6, 9, 25, 30 y 50 h); tratamiento (aplicación de glifosato y control) y las interacciones dobles y triple sobre el crecimiento

\begin{tabular}{|c|c|c|c|}
\hline Fuente de variación & GL & $\mathrm{CM}$ & $\mathrm{P}$ \\
\hline Biotipo & 1 & 87,55 & 0,005 \\
\hline Horas post-aplicación & 5 & 1498,53 & $<0,001$ \\
\hline Tratamiento & 1 & 322,42 & $<0,001$ \\
\hline Biotipo x Horas post-aplicación & 5 & 11,86 & 0,372 \\
\hline Biotipo $\mathrm{x}$ Tratamiento & 1 & 209,35 & $<0,001$ \\
\hline Horas post-aplicación $\mathrm{x}$ Tratamiento & 5 & 48,72 & 0,001 \\
\hline Biotipo $\mathrm{x}$ Horas post-aplicación $\mathrm{x}$ Tratamiento & 5 & 43,53 & 0,002 \\
\hline Error & 96 & 10,9 & \\
\hline
\end{tabular}

$\mathrm{GL}$ = grados de libertad; $\mathrm{CM}=$ cuadrados medios; $\mathrm{p}$ = valor de la prueba de $\mathrm{F}$.

Roso \& Vidal (2010) sugieren la posibilidad de que proteínas fosfato-transportadoras estén implicadas en mecanismos de reducida
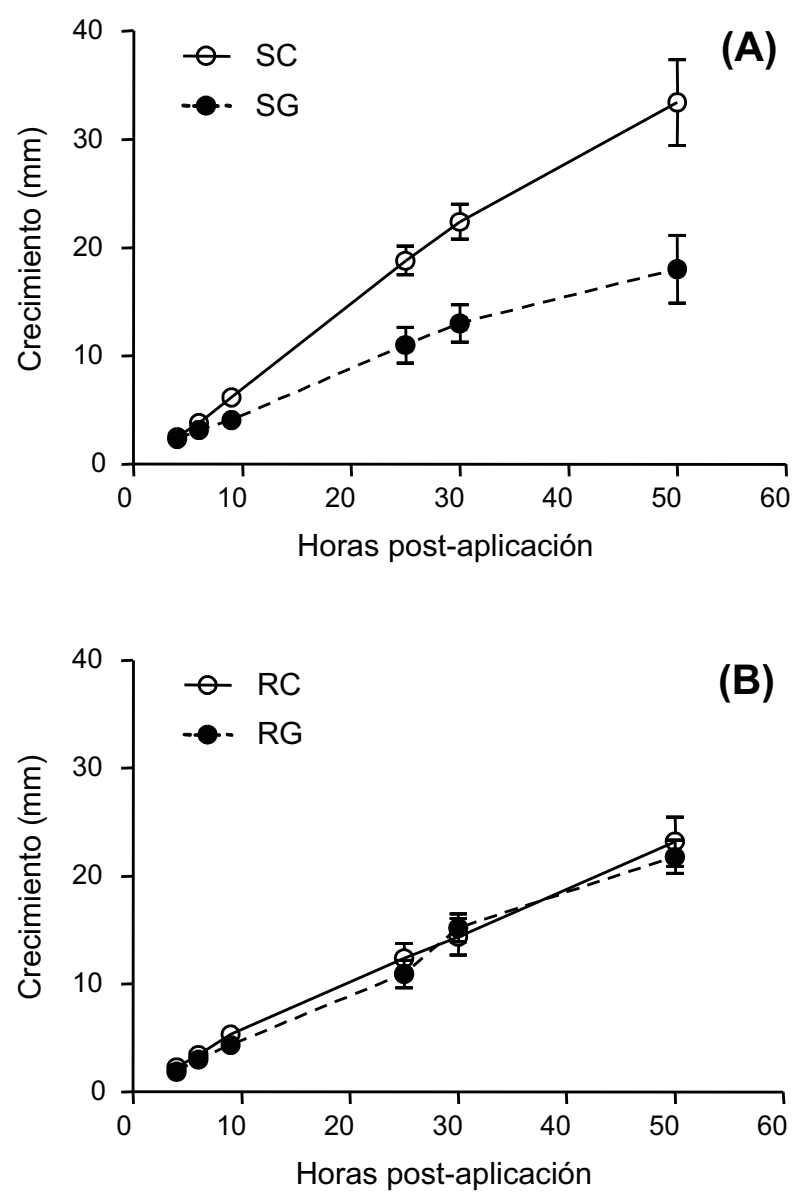

Figura 1 - Efecto del glifosato sobre el crecimiento longitudinal de hojas. A) Biotipo susceptible control (SC) y tratado con glifosato (SG); B) Biotipo resistente control (RC) y tratado con glifosato (RG). Se presentan los promedios \pm el error estándar de la media. translocación o vacuolización de glifosato. En cualquier caso, se espera que el efecto del herbicida sobre la actividad meristemática sea reducido o nulo en plantas resistentes, como ha sucedido en nuestros materiales.

\section{Efectos sobre la acumulación de azúcares libres}

Determinación de azúcares totales: La concentración de azúcares totales varió significativamente por el efecto del tratamiento con glifosato, así como con los días post-aplicación, siendo la interacción de ambas estadísticamente significativa (Tabla 2). A partir del análisis de varianza, se detectaron otras interacciones dobles de significancia estadística. Los biotipos interactuaron con el tratamiento, manifestándose diferencias altamente significativas en la acumulación de azúcares totales (Tabla 2).

A un DPA se hallaron diferencias significativas $(p<0,05)$ entre plantas del biotipo susceptible tratadas con glifosato y sus controles, mostrando una tendencia al incremento de azúcares totales libres producto de la aplicación del herbicida. A los 2 DPA y en las determinaciones consecutivas, el incremento de azúcares totales en hoja se acentuó bajo el tratamiento con glifosato, diferenciándose significativamente del control $(\mathrm{p}<0,05)$ (Figura 2). El glifosato incrementó la concentración de azúcares totales en el biotipo susceptible en un $90 \%$ a 1 DPA; posteriormente, estas diferencias fueron del 124\%, 180\% y $100 \%$ a los 2, 3 y 5 DPA, respectivamente (Figura 2). En forma coincidente, Ulanov et al. (2009) señalaron que el tratamiento con glifosato 
Tabla 2 - Efectos del biotipo (susceptible y resistente), los días post-aplicación (1, 2, 3 y 5 días), tratamiento (aplicación de glifosato y control) y las interacciones dobles y triple sobre la acumulación de azúcares totales libres en hojas

\begin{tabular}{|l|c|c|c|}
\hline \multicolumn{1}{|c|}{ Fuente de variación } & GL & CM & P \\
\hline Biotipo & 1 & $1,792 \times 107$ & 0,110 \\
\hline Días post-aplicación & 3 & $3,225 \times 108$ & $<0,001$ \\
\hline Tratamiento & 1 & $5,109 \times 108$ & $<0,001$ \\
\hline Biotipo x Días post-aplicación & 3 & $2,250 \times 107$ & 0,026 \\
\hline Biotipo x Tratamiento & 1 & $1,473 \times 108$ & $<0,001$ \\
\hline Días post-aplicación x Tratamiento & 3 & $1,964 \times 107$ & 0,043 \\
\hline Biotipo x Días post-aplicación x Tratamiento & 3 & $8,176 \times 106$ & 0,319 \\
\hline Error & 64 & $6,853 \times 106$ & \\
\hline
\end{tabular}

$\mathrm{GL}=$ grados de libertad; $\mathrm{CM}=$ cuadrados medios; $\mathrm{p}=$ valor de la prueba de $\mathrm{F}$.
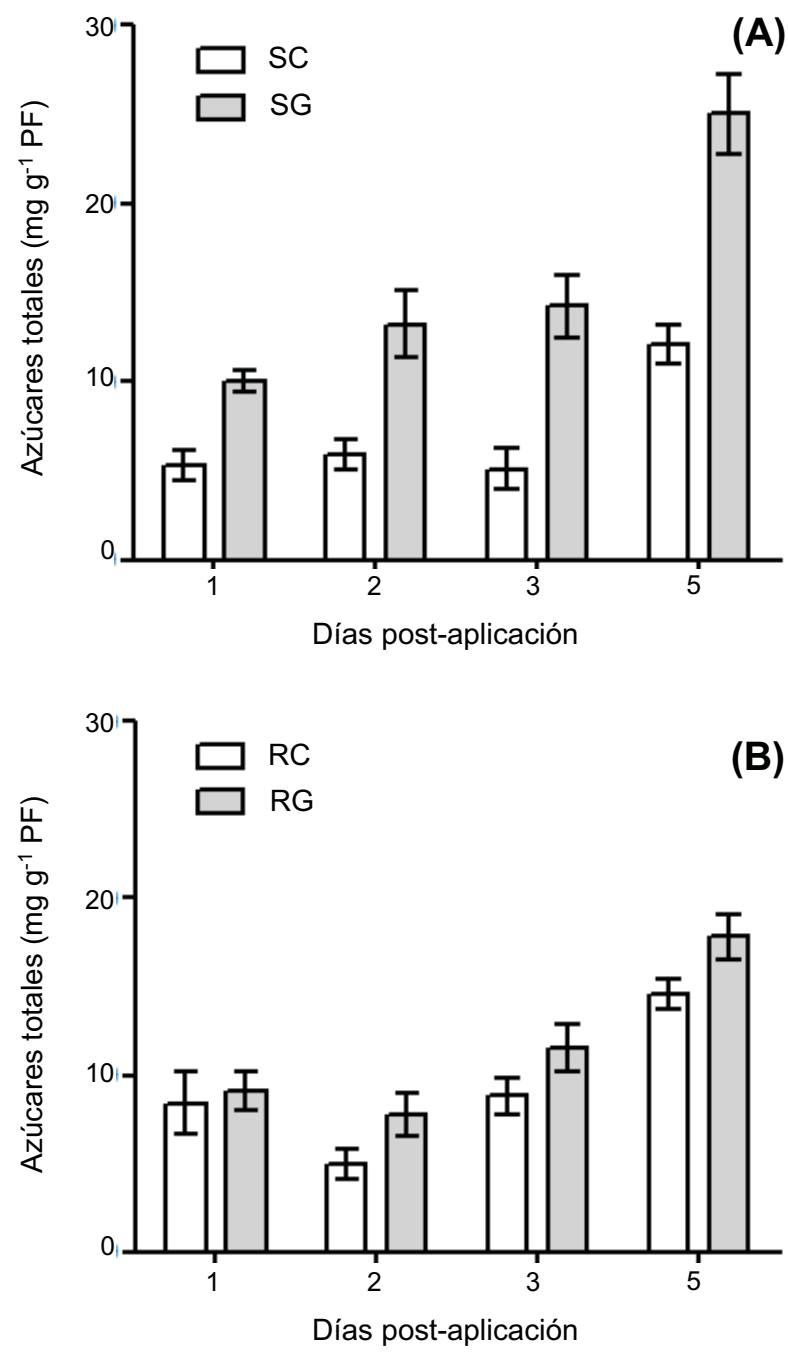

Figura 2 - Acumulación foliar de azúcares totales libres expresada sobre peso fresco (PF) durante post-aplicación: A) Biotipo susceptible control (SC) y tratado con glifosato (SG); B) Biotipo resistente control (RC) y tratado con glifosato (RG). Se presentan los promedios \pm el error estándar de la media. condiciona la acumulación de azúcares totales en tejidos de Zea mays cultivados in vitro, e indicaron que los principales carbohidratos responsables por los elevados tenores de azúcares totales son sacarosa, glucosa, sorbosa, fructosa y galactosa.

El análisis de los resultados indica que el aumento en los niveles de azúcares libres podria ser una consecuencia de la disminución de la tasa del crecimiento. Geiger y Bestman (1990), al estudiar el problema de la "autolimitación" del traslado de glifosato, presentaron la posible relación entre la caída en la demanda de sacarosa, debido a la disminución de la síntesis proteica en tejidos en crecimiento, y sus efectos sobre la disminución del traslado del herbicida.

Mengel \&Kirkby (2001) indicaron que la demanda de azúcares de un destino está condicionada por su actividad metabólica. En concordancia, los efectos del glifosato sobre la acumulación de carbohidratos totales no serian detectados en plantas resistentes, debido a que el mecanismo de resistencia puesto en juego le permite mantener la tasa de crecimiento y no alterar la demanda de azúcares en las fuentes.

Determinación de azúcares reductores: Hubo diferencias altamente significativas en los azúcares reductores en los distintos lapsos de tiempo y entre los tratamientos (Tabla 3). Al igual que en el caso de los azúcares totales, hubo diferencias altamente significativas entre ambos biotipos a lo largo del tiempo y frente a los tratamientos. Al mismo tiempo, el contenido de azúcares reductores fue 
Tabla 3 - Análisis de la varianza de los efectos del biotipo (susceptible y resistente), los días post-aplicación (1, 2, 3 y 5 días), tratamiento (aplicación de glifosato y control) y las interacciones dobles y triple sobre la acumulación de azúcares reductores libres en hojas

\begin{tabular}{|c|c|c|c|}
\hline Fuente de variación & GL & $\mathrm{CM}$ & $\mathrm{P}$ \\
\hline Biotipo & 1 & $1,886 \times 107$ & 0,003 \\
\hline Días post-aplicación & 3 & $5,424 \times 107$ & $<0,001$ \\
\hline Tratamiento & 1 & $8,300 \times 107$ & $<0,001$ \\
\hline Biotipo x Días post-aplicación & 3 & $9,863 \times 106$ & 0,003 \\
\hline Biotipo $\mathrm{x}$ Tratamiento & 1 & $5,926 \times 107$ & $<0,001$ \\
\hline Días post-aplicación $\mathrm{x}$ Tratamiento & 3 & $6,201 \times 106$ & 0,033 \\
\hline Biotipo x Días post-aplicación x Tratamiento & 3 & $6,439 \times 106$ & 0,029 \\
\hline Error & 64 & $2,013 \times 106$ & \\
\hline
\end{tabular}

$\mathrm{GL}$ = grados de libertad; $\mathrm{CM}=$ cuadrados medios; $\mathrm{p}=$ valor de la prueba de $\mathrm{F}$.

significativamente afectado por el herbicida en los distintos DPA. Finalmente, la interacción triple entre las tres fuentes de variación: biotipo x dias post-aplicación xtratamiento fue estadísticamente significativa. Hubo un comportamiento diferencial entre los biotipos a lo largo del tiempo con y sin herbicida (Tabla 3). A partir de los 2 DPA, la concentración de azúcares reductores fue afectada significativamente por el herbicida con relación al control (Figura 3). Esos incrementos de azúcares reductores, en promedio, fueron el $130 \%$ superiores a los valores determinados en las plantas control. Los resultados de Ulanov et al. (2009) previamente citados, respecto a la acumulación de azúcares reductores como glucosa, sorbosa, fructosa y galactosa en respuesta al glifosato, concuerdan con los obtenidos en el presente ensayo.

En contraste con los resultados del biotipo susceptible, las plantas resistentes no mostraron incrementos significativos en los niveles de azúcares reductores producto de la aplicación del herbicida (Figura 3). Anteriormente, Pline et al. (2003) evaluaron el efecto del glifosato sobre la acumulación de fructosa y glucosa en hojas de algodón resistente al herbicida y, en coincidencia a los resultados obtenidos, no se detectaron diferencias entre plantas tratadas y los controles sin herbicida. Los resultados de la determinación de azúcares reductores coinciden con los referidos a la acumulación de azúcares totales y su relación con la insensibilidad al tratamiento con glifosato de los tejidos en crecimiento.
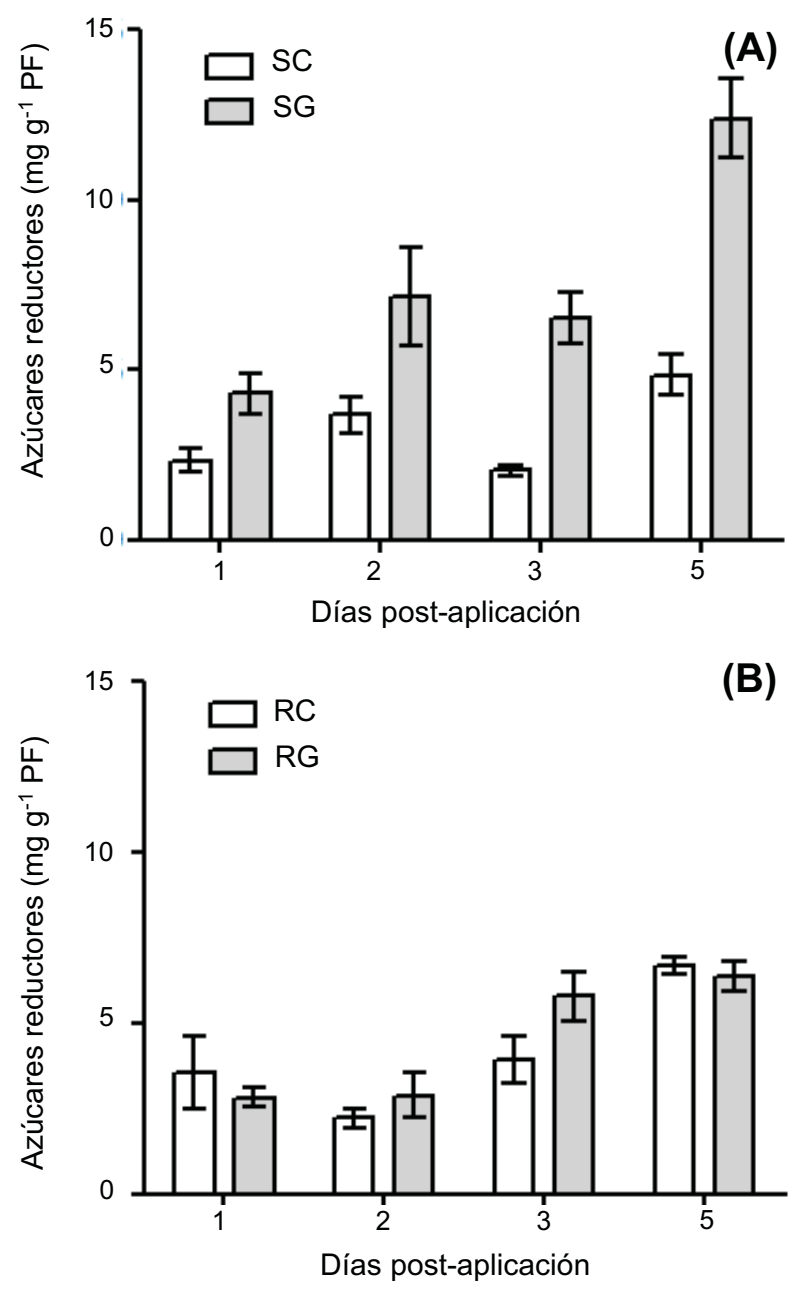

Figura 3 - Acumulación foliar de azúcares reductores libres expresada sobre peso fresco (PF) durante post-aplicación: A) Biotipo susceptible control (SC) y tratado con glifosato (SG); B) Biotipo resistente control (RC) y tratado con glifosato (RG). Se presentan los promedios \pm el error estándar de la media.

Planta Daninha, Viçosa-MG, v. 30, n. 1, p. 155-164, 2012 
Estimación de azúcares no reductores: La interacción entre los biotipos y los tratamientos explica la variación de azúcares no reductores $(\mathrm{p}<0,05)$. A esta fuente de variación se le agrega el efecto aditivo de los días postaplicación ( $\mathrm{p}<0,001)$ (Tabla 4).

En el biotipo susceptible, el glifosato afectó significativamente los niveles de azúcares no reductores desde los 2 DPA en adelante (Figura 4). El herbicida provocó un incremento en la acumulación de estos carbohidratos del 90\% ( \pm 40\% de desvío estándar), en promedio, para todos los días post-aplicación.

Considerando el biotipo resistente, el glifosato frente a los controles sólo provocó una diferencia significativa a los 5 DPA (Figura 4). En esta evaluación, el efecto del herbicida causó un incremento de azúcares no reductores del 45\%. Esta diferencia no se reflejó en las determinaciones realizadas sobre azúcares reductores, lo que permite sugerir que el herbicida podría provocar una disminución de la demanda de sacarosa desde distintos órganos. Este efecto sería relativamente tardio comparado con plantas susceptibles y no altera significativamente la sintesis de monosacáridos. Su et al. (1992) reportaron que el glifosato reduce significativamente la actividad de enzimas invertasas ácidas, responsable por la hidrólisis de sacarosa, conduciendo a un incremento de los tenores de este disacárido.

En plantas susceptibles, el glifosato se transloca rápidamente a tejidos meristemáticos y causa una severa inhibición del crecimiento, como fue demostrado en el primer experimento. De acuerdo con Nafziger $\&$ Slife (1983), el incremento en los niveles de carbohidratos solubles sugiere que la inhibición del crecimiento sucedería antes de la inhibición de la producción de fotosintatos. Azcón Bieto (1983) demostró que tratamientos que reducen la tasa de traslado de asimilados promueven una caída de la fijación de $\mathrm{CO}_{2}, \mathrm{y}$ esa inhibición de la fotosintesis se correlaciona con la acumulación de asimilados en la hoja. Coincidiendo con ello, Ribeiro et al. (2008) hallaron que el glifosato provocó caídas en la fotosintesis neta de plantas susceptibles de Lolium multiflorum y, conduciendo a valores de la mitad de las tasas registradas en plantas resistentes a 48 HPA.

Fuchs et al. (2002) demostraron que los niveles de 3-fosfoglicerato y ribulosa bifosfato carboxilasa son sensibles al tratamiento con glifosato y caen significativamente luego de las 24 h de aplicado. Los incrementos de azúcares libres en las hojas serian responsables por la inhibición en la regeneración de ribulosa1,5-bifosfato. Esto limitaría la fijación de $\mathrm{CO}_{2}$ (Azcón Bieto, 1983). A ese efecto se sumaría la pérdida de eritrosa-4-fosfato del ciclo de Calvin vía la ruta del ácido shikímico, por la desregulación provocada por el glifosato al inhibir la EPSPs (Duke \& Powles, 2008). Esto limitaría aún más la regeneración de ribulosa-1,5-bifosfato y explicaría los efectos del herbicida sobre la caída en el intercambio neto de carbono previamente citado (Ribeiro et al., 2008; Olesen \& Cedergreen, 2010).

El análisis de los resultados corrobora la hipótesis planteada de que la inhibición del crecimiento provocada por el glifosato en

Tabla 4 - Efectos del biotipo (susceptible y resistente), los días post-aplicación (1, 2, 3 y 5 días), tratamiento (aplicación de glifosato y control) y las interacciones dobles y triple sobre la acumulación de azúcares no reductores libres en hojas

\begin{tabular}{|l|r|r|r|}
\hline \multicolumn{1}{|c|}{ Fuente de variación } & GL & CM & P \\
\hline Biotipo & 1 & $2,842 \times 10^{5}$ & 0,797 \\
\hline Días post-aplicación & 3 & $1,107 \times 10^{8}$ & $<0,001$ \\
\hline Tratamiento & 1 & $1,668 \times 10^{8}$ & $<0,001$ \\
\hline Biotipo x Días post-aplicación & 3 & $2,954 \times 10^{6}$ & 0,561 \\
\hline Biotipo x Tratamiento & 1 & $1,933 \times 10^{7}$ & 0,037 \\
\hline Días post-aplicación x Tratamiento & 3 & $2,954 \times 10^{6}$ & 0,562 \\
\hline Biotipo x Días post-aplicación x Tratamiento & 3 & $4,407 \times 10^{6}$ & 0,386 \\
\hline Error & 64 & $4,285 \times 10^{6}$ & -3 \\
\hline
\end{tabular}

$\mathrm{GL}$ = grados de libertad; $\mathrm{CM}=$ cuadrados medios; $\mathrm{p}=$ valor de la prueba de $\mathrm{F}$. 

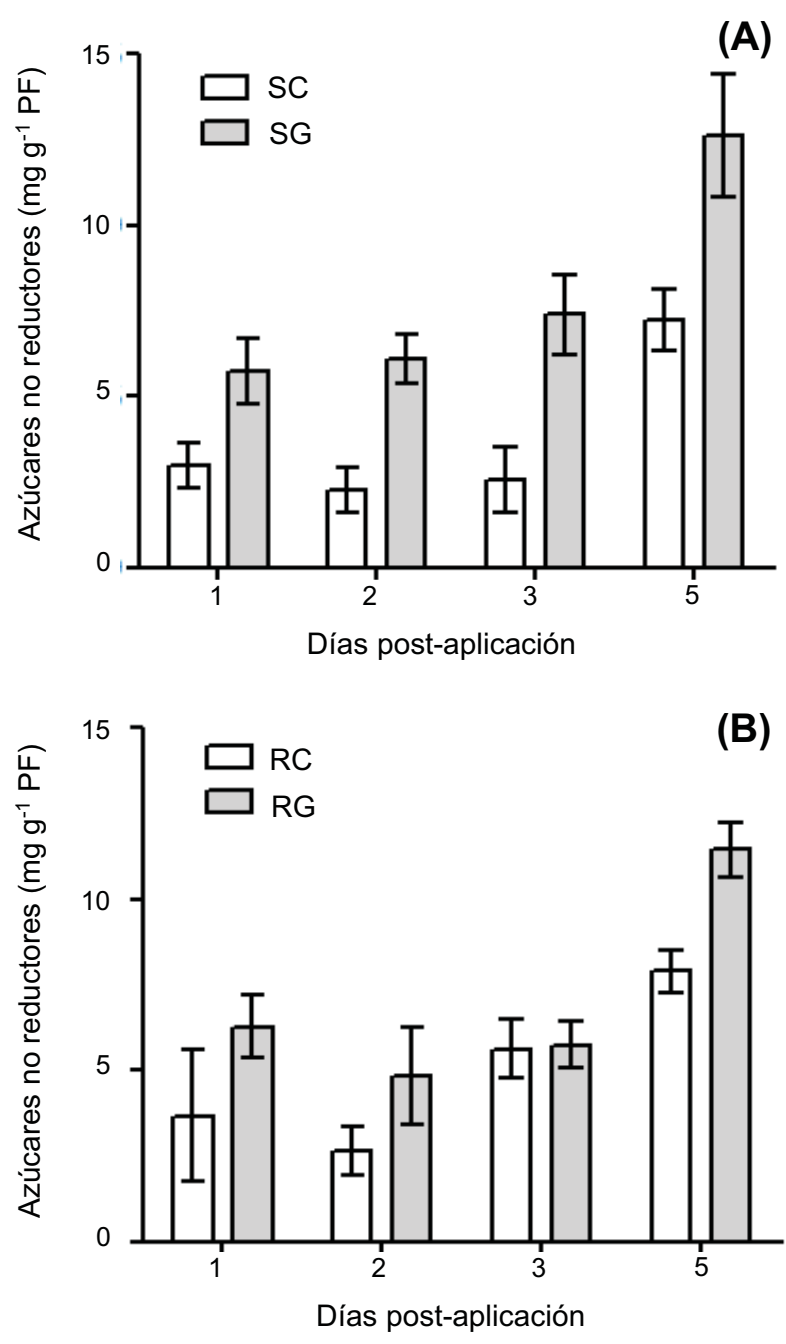

Figura 4 - Acumulación foliar de azúcares no reductores libres expresada sobre peso fresco (PF) durante postaplicación: A) Biotipo susceptible control (SC) y tratado con glifosato (SG); B) Biotipo resistente control (RC) y tratado con glifosato (RG). Se presentan los promedios \pm el error estándar de la media.

plantas susceptibles no es dependiente de la limitación del traslado de fotoasimilados desde la parte aérea. Por tanto, la acumulación de azúcares libres en hojas de plantas susceptibles sería una consecuencia de la caída en la tasa de crecimiento generada por el glifosato. Estos efectos se ven limitados en el biotipo resistente, donde no se detectó inhibición del crecimiento.

\section{LITERATURA CITADA}

AZCON BIETO, J. Inhibition of photosynthesis by carbohydrates in wheat leaves. Plant Physiol., v. 73, n. 3, p. 681-686, 1983.
BAERSON S. R. et al. Investigating the mechanism of glyphosate resistance in rigid ryegrass (Lolium rigidum). Weed Sci., v. 50, n. 6, p. 721-730, 2002.

BROMILOW, R. H. et al. Physicochemical aspects of phloem translocation of herbicides. Weed Sci., v. 38, n. 3, p. 305-314, 1990.

DEVINE, M. D.; HALL, L. M. Implications of sucrose translocation mechanisms for the translocation of herbicides. Weed Sci., v. 38, n. 3, p. 299-304, 1990.

DUKE, S. O.; POWLES, S. B. Glyphosate: a once-in-acentury herbicide. Pest Manag. Sci., v. 64, n. 4, p. 319-325, 2008.

EL-TAYEB, M. A.; ZAKI, H. Cytophysiological response of Vicia faba to a glyphosate-based herbicide. American-Eur. J. Agron., v. 2, n. 1, p. 168-175, 2009.

FRANZ, J. E. et al. Glyphosate: A unique and global herbicide. Washington, DC: American Chemical Society, 1997. 653 p. (ACS Monograph, 189)

FUCHS, M. A. et al. Mechanisms of glyphosate toxicity in velvetleaf (Abutilon theophrasti Medikus). Pest. Biochem. Physiol., v. 74, n. 1, p. 27-39, 2002.

GEIGER, D. R.; BESTMAN, H. D. Self-limitation of herbicide mobility by phytotoxic action. Weed Sci., v. 38, n. 3, p. 324-329, 1990.

GEIGER, D. et al. Causes of self-limited translocation of glyphosate in Beta vulgaris plants. Pest. Biochem. Physiol., v. 64, n. 1, p. 124-133, 1999.

MENGEL, K.; KIRKBY, E. A. Principles of plant

nutrition. 5.ed. London: Kluwer Academic Publishers, 2001. 849 p.

NAFZIGER, E. D.; SLIFE, F. W. Physiological response of common cocklebur (Xanthium pensylvanicum) to glyphosate. Weed Sci., v. 31, n. 6, p. 874-878, 1983.

OLESEN, C. F.; CEDERGREEN, N. Glyphosate uncouples gas exchange and chlorophyll fluorescence. Pest Manag. Sci., v. 66, n. 5, p. 536-542, 2010.

OWEN, M. Weed species shifts in glyphosate-resistant crops. Pest Manag. Sci., v. 64, n. 4, p. 377-387, 2008.

PEREZ, A.; KOGAN, M. Glyphosate-resistant Lolium multiflorum in Chilean orchards. Weed Res., v. 43, n. 1, p. 12-19, 2003.

PEREZ-JONES, A. et al. Investigating the mechanisms of glyphosate resistance in Lolium multiflorum. Planta, v. 226, n. 2, p. 395-404, 2007. 
PLINE, W. A. et al. Physiological and morphological response of glyphosate-resistant and non-glyphosate-resistant cotton seedlings to root-absorbed glyphosate. Pest. Biochem.

Physiol., v. 73, n. 1, p. 48-58, 2002.

RIBEIRO, D. N. et al. Rapid assays for detection of glyphosate-resistant Lolium spp. J. Plant Dis. Protec., v. 21, n. 1, p. 95-100, 2008.

ROMAN, E. S. et al. Resistance of Italian ryegrass (Lolium multiflorum) to glyphosate. Planta Daninha, v. 22, n. 2, p. 301-306, 2004.

ROSO, A. C.; VIDAL, R. A. A modified phosphate-carrier protein theory is proposed as a non-target site mechanism for glyphosate resistance in weeds. Planta Daninha, v. 28, p. 1175-1185, 2010. (Numero Especial)

SCHÖNBRUN, E. et al. Interaction of the herbicide glyphosate with its target enzyme 5-enolpyruvylshikimate 3-phosphate synthase in atomic detail. Proc. Nat. Acad. Sci., v. 98, n. 4, p. 1376-1380, 2001.

SERVAITES, J. et al. Glyphosate effects on carbon assimilation, ribulose biphosphate carboxylase activity, and metabolite levels in sugar beet leaves. Plant Physiol., v. 85, n. 2, p. 370-374, 1987.

SHANER, D. L. Role of translocation as a mechanism of resistance to glyphosate. Weed Sci., v. 57, n. 1, p. 118-123, 2009.

SU, L. Y. et al. The relationship of glyphosate treatment to sugar metabolism in sugarcane: new physiological insights. J. Plant Physiol., v. 140, n. 1, p. 168-173, 1992.
ULANOV, A. et al. Metabolic effects of glyphosate change the capacity of maize culture to regenerate plants. J. Plant Physiol., v. 166, n. 9, p. 978-987, 2009.

VARGAS, L. Identification of glyphosate-resistant ryegrass (Lolium multiflorum) biotypes in apple orchards.

Planta Daninha, v. 22, n. 4, p. 617-622, 2004.

VIGNA, M. R. et al. Estudios de curvas Dosis-respuesta de poblaciones de Lolium multiflorum a glifosato en el SO de Buenos Aires, Argentina. En: CONGRESO LATINOAMERICANO DE MALEZAS, 18., 2008, Ouro Preto. Anais... Ouro Preto: 2008. CD-ROM.

VILA-AIUB, M. et al. Glyphosate-resistant weeds of South American cropping systems: an overview. Pest Manag. Sci., v. 64, n. 4, p. 366-371, 2008.

WAKELIN, A. M.; PRESTON, C. A target-site mutation is present in a glyphosate-resistant Lolium rigidum population. Weed Res., v. 46, n. 5, p. 432-440, 2006.

WOODBURN, A. T. Glyphosate: production, pricing and use worldwide. Pest Manag. Sci., v. 56, n. 4, p. 309-312, 2000.

YAMADA, T. et al. Glyphosate interactions with physiology, nutrition, and diseases of plants: threats to agricultural sustainability? Europ. J. Agron., v. 31, n. 3, p. 111-113, 2009.

YANNICCARI, M. et al. Glyphosate resistance in perennial ryegrass (Lolium perenne L.) from Argentina. Crop Protec., v. 32, n. 1, p. 12-16, 2012. 